\title{
Development of Object- Oriented Tools for the Numerical Solution of Reactive Flow
}

D.W. Schwendeman

September 30, 2000

U.S. Department of Energy

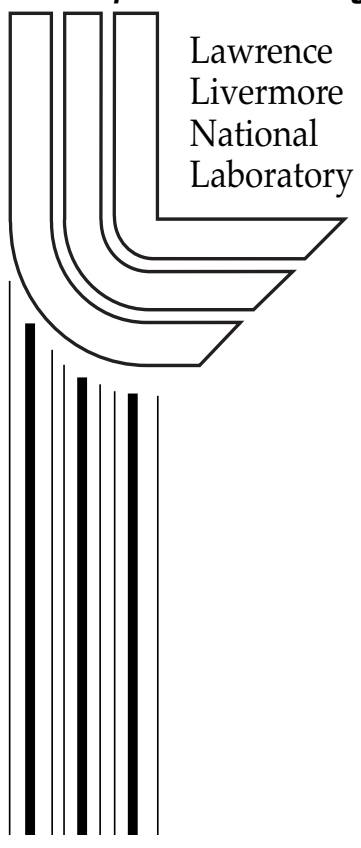




\section{DISCLAIMER}

This document was prepared as an account of work sponsored by an agency of the United States Government. Neither the United States Government nor the University of California nor any of their employees, makes any warranty, express or implied, or assumes any legal liability or responsibility for the accuracy, completeness, or usefulness of any information, apparatus, product, or process disclosed, or represents that its use would not infringe privately owned rights. Reference herein to any specific commercial product, process, or service by trade name, trademark, manufacturer, or otherwise, does not necessarily constitute or imply its endorsement, recommendation, or favoring by the United States Government or the University of California. The views and opinions of authors expressed herein do not necessarily state or reflect those of the United States Government or the University of California, and shall not be used for advertising or product endorsement purposes.

Work performed under the auspices of the U. S. Department of Energy by the University of California Lawrence Livermore National Laboratory under Contract W-7405-Eng-48. 
Rensselaer Polytechnic Institute

Department of Mathematical Sciences

Troy, New York 12180

\section{Final Report for \\ "Development of Object-Oriented Tools for the Numerical Solution of Reactive Flow"}

Submitted by:

Donald W. Schwendeman

Associate Professor of Applied Mathematics

FINAL REPORT

For the period ending September 30, 2000.

Prepared for:

University of California

Lawrence Livermore National Laboratory

Attn: Jill Dunaway

P.O. Box 808, L-561

Livermore, CA 94551

Under:

LLNL subcontract number B503621

RPI contract number A90104 


\title{
Final Report on
}

\author{
LLNL Subcontract B503621 \\ Development of Object-Oriented Tools \\ for the Numerical Solution of Reactive Flow
}

\author{
Donald W. Schwendeman
}

\section{Project Overview}

The primary work carried out under the subcontract involved the development of object-oriented application software, within the Overture framework of codes, for the numerical simulation of high speed reactive flow. The mathematical model on which the software is based is the reactive Euler equations. The implementation of this model is fairly general and allows for multiple reacting species and reaction rates and a general equation of state with the aim of being able to simulate experimentally observed phenomena in gas or solid explosives. The software is part of the OverBlown package of fluids codes, developed and maintained by Bill Henshaw and the Overture team at CASC. It uses overlapping grids in order to handle general domains and the $\mathrm{A}++/ \mathrm{P}++$ array class library (developed by Dan Quinlan and the Overture team) which allows parallel processing. An implementation of a patch grid-type adaptive mesh refinement (AMR) scheme for the code was initiated, but not completed under the current subcontract. The software has been carefully tested for accuracy using existing codes written previously by the author, and it has been used to compute the evolution to detonation of reactive samples subject to various initial conditions and within various confinement geometries.

Work on a number of smaller projects has also taken place. These include methods for conservative interpolation on general curvilinear grids, methods for characteristic interpolation, and developing software tools for numerical quadrature on overlapping grids.

\section{Activities Supported in Part by the Subcontract}

Results of the subcontract work were presented at a minisymposium organized by David Brown and the author at the First SIAM Conference on Computational Science and Engineering, September 21-24, 2000, in Washington, DC.

\section{Project Highlights}

The main work involved the development of software for the numerical solution of the reactive Euler equations. These equations are a system of nonlinear, hyperbolic conservation laws that, in two space dimensions, have the form

$$
u_{t}+f(u)_{x}+g(u)_{y}=h(u),
$$


where $(x, y)$ are Cartesian coordinates, $t$ is time, $u$ is set of state variables, $(f, g)$ are fluxes, and $h$ is a source term. The latter are given by

$$
u=\left[\begin{array}{c}
\rho \\
\rho u \\
\rho v \\
\rho E \\
\rho \lambda
\end{array}\right] \quad f=\left[\begin{array}{c}
\rho u \\
\rho u^{2}+p \\
\rho u v \\
u(\rho E+p) \\
\rho u \lambda
\end{array}\right] \quad g=\left[\begin{array}{c}
\rho v \\
\rho u v \\
\rho v^{2}+p \\
v(\rho E+p) \\
\rho v \lambda
\end{array}\right] \quad h=\left[\begin{array}{c}
0 \\
0 \\
0 \\
0 \\
\rho R
\end{array}\right]
$$

where

$$
\begin{aligned}
\rho & =\text { density, } & (u, v) & =\text { velocity } \\
p & =\text { pressure, } & E & =\text { total energy } \\
\lambda & =m \text { mass fractions, } & R & =m \text { reaction rates. }
\end{aligned}
$$

The total energy is given by

$$
E=e+\frac{1}{2}\left(u^{2}+v^{2}\right)-\sum_{k=1}^{m} \lambda_{k} Q_{k},
$$

where $Q_{k}$ is the heat release for species $k$ and the internal energy is related to density and pressure by a general equation of state, $e=e(\rho, p)$. For a given simulation, these equations are to be solved on a given domain subject to various initial conditions and boundary conditions.

A numerical method of solution was implemented for the reactive Euler equations. Within the Overture framework [1], the numerical method discretizes the equations on a set of overlapping, structured grids that cover the domain of interest. The software package Ogen [2] is used to generate the overlapping grid and provide geometric mapping information for each curvilinear component grid and information concerning the communication of the solution between grids in the overlap region. This information was considered to be given and thus the main task was to implement the numerical method for an individual component grid and then let the exisiting software, OverBlown [3, 4], handle the surrounding numerical details (such as interpolation between component grids, the application of boundary conditions, time stepping, graphical interface, and more). The use and modification of existing software was essential for the development of the software within the 9 month period of the subcontract.

For an individual component grid, an unsplit Godunov-type, finite volume method was used to solve the equations numerically. A mapping is given (by Ogen) from physical coordinates $(x, y)$ to computational coordinates $(r, s)$. In terms of the computational coordinates, the equations become

$$
U_{t}+F(U)_{r}+G(U)_{s}=H(U),
$$

where $U, F, G$, and $H$ are given in terms of $u, f, g$, and $h$ and derivatives of the mapping and the grid velocity. On a grid $\left(r_{j}, s_{k}\right)$ with mesh spacings $\Delta r$ and $\Delta s$, the basic discretization is

$$
\tilde{U}_{j, k}(t+\Delta t)=\tilde{U}_{j, k}(t)+\Delta t \dot{U}_{j, k}(t),
$$


where the numerical solution $\tilde{U}_{j, k}(t)$ at a time $t$ is advanced to time $t+\Delta t$ using the numerical derivative

$$
\dot{U}_{j, k}=\tilde{H}_{j, k}-\frac{\tilde{F}_{j, k}-\tilde{F}_{j-1, k}}{\Delta r}-\frac{\tilde{G}_{j, k}-\tilde{G}_{j, k-1}}{\Delta s} .
$$

In this expression, the numerical fluxes $\tilde{F}$ and $\tilde{G}$ are obtained using a second-order extension of Godonov's method [5] and $\tilde{H}$ is a numerical source term. The approximate Riemann solver is a Roe-type method extended to handle a general equation of state [6]. The numerical source term is obtained by advancing the $m$ species variables from $t$ to $t+\Delta t$ (while holding the remaining gas dynamic variables fixed) according to the $m$ ordinary differential equations (ODEs)

$$
\frac{d \lambda_{k}}{d t}=R_{k}(U), \quad k=1, \ldots, m
$$

These ODEs are solved numerically for each grid cell using a Runge-Kutta, variable time step, error control algorithm. This is done to ensure that the (possibly) stiff rate equations are solved accurately for a CFL time step, $\Delta t$.

Several example calculations were run to test the software and to study the evolution to detonation of a reactive sample at critical conditions subject to gradients in temperature. The physical motivation for these calculations was to examine the effects of small nonuniformities in the initial state and of the confinement geometry on the severity of the reaction in terms of peak temperatures and pressures.

Several calculations were run for a sample with an initial state composed of pure fuel (assuming a single-species problem, $m=1$ ) at rest with a uniform pressure and small gradient in temperature. For this initial state, the variables are

$$
u=v=\lambda=0, \quad p=1, \quad T=1-\alpha \theta(x, y) .
$$

Here, $T=p / \rho$ is (dimensionless) temperature, $\alpha$ is a small parameter, and $\theta(x, y)$ is an $O(1)$ function that specifies the form of the gradient in temperature. A simple choice is

$$
\theta(x, y)=\sqrt{\left(x-x_{0}\right)^{2}+\left(y-y_{0}\right)^{2}}
$$

where $\left(x_{0}, y_{0}\right)$ is the location of an initial maximum in temperature, i.e. an initial "hot spot". A suitable dimensionless reaction rate was taken to be

$$
R=\sigma(1-\lambda) \exp \left[\frac{1}{\epsilon}\left(1-\frac{1}{T}\right)\right],
$$

where $\sigma$ is a prefactor and $1 / \epsilon$ is a dimensionless activation energy. For highly state sensitive explosives, $\epsilon$ is a small parameter. The prefactor $\sigma$ determines the time scale for the reaction. If $\sigma=\epsilon$ and an ideal gas law, $e=p /(\gamma-1) \rho$, is assumed, then in the limit of very small $\epsilon$ the dimensionless blow-up time is $1 / Q(\gamma-1)=O(1)$ for a uniform, constant density explosion (i.e. the case when $\alpha=0$ in the initial state given above). 


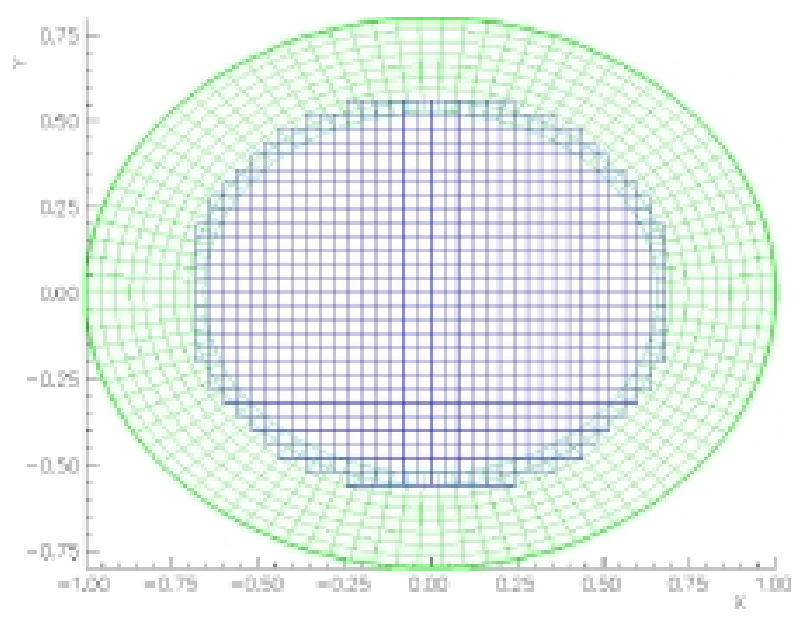

Figure 1: Composite overlapping grid for hot-spot calculations

The interesting evolution from the initial state described above occurs when $\alpha$, the gradient parameter for temperature, is $O(\epsilon)$. If $\alpha$ is too small, then an explosion takes place as a "phase wave" in which a reaction front is born from the initial hotspot location but travels too fast for significant acoustic information to propagate and strengthen the wave to form a detonation. On the other side, if $\alpha$ is too large, then a local explosion from the initial hot spot does not create a large enough response to initiate significant reaction in the cold neighboring material. In this case, a detonation would not occur. In between these extremes, there is a range of values for $\alpha$ for which the initial gradient leads to a constructive interplay between chemical activity and acoustic propagation resulting in the evolution to a shock-supported detonation. This event leads to high peak temperatures and pressures at the leading edge of the detonation which are enhansed still further by interactions with a solid confinement. The initial location of the hot spot, the size of the initial gradient (modeled by $\alpha$ ), and the geometry of the confinement all play a role in the severity of the event.

As an example, the plots below, generated with the software visualization package PlotStuff [7], illustrate one case in which a detonation is generated from an initial hot spot and interacts with a solid elliptical confinement. The parameters for this calculation are

$$
\alpha=0.075, \quad\left(x_{0}, y_{0}\right)=(0.2,0), \quad \sigma=0.05, \quad \epsilon=0.06, \quad Q=3.0,
$$

and the solid wall has the shape of an ellipse given by $x^{2}+(y / 0.8)^{2}=1$. A coarse version of the overlapping grid used for this calculation is shown in Figure 1. The actual grid used a $56 \times 753$ boundary grid (green grid) and a $289 \times 241$ background grid (blue grid). Figure 2 illustrates the evolution of the temperature from the initial state. By $t=1.5$ (upper left frame), the initial hot spot has grown to a stage where significant reaction has begun. The peak value of $\lambda$ at the this time is approximately $1 / 2$. At later times, a shock-supported detonation forms. The detonation propagates 


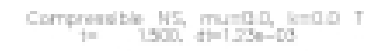

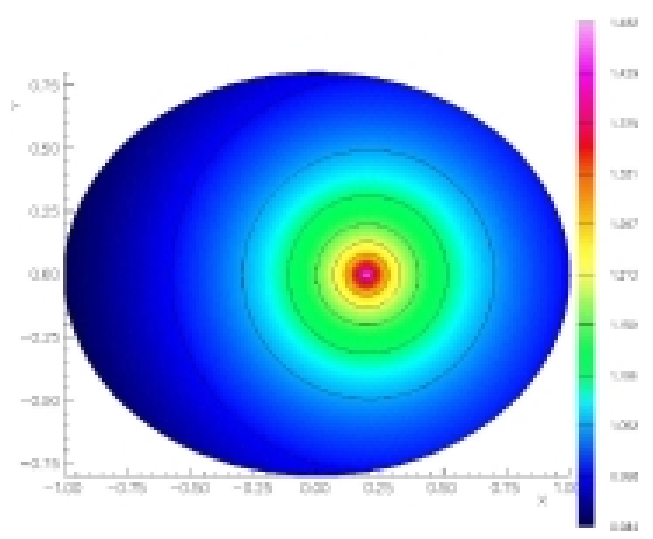

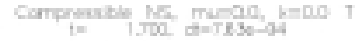

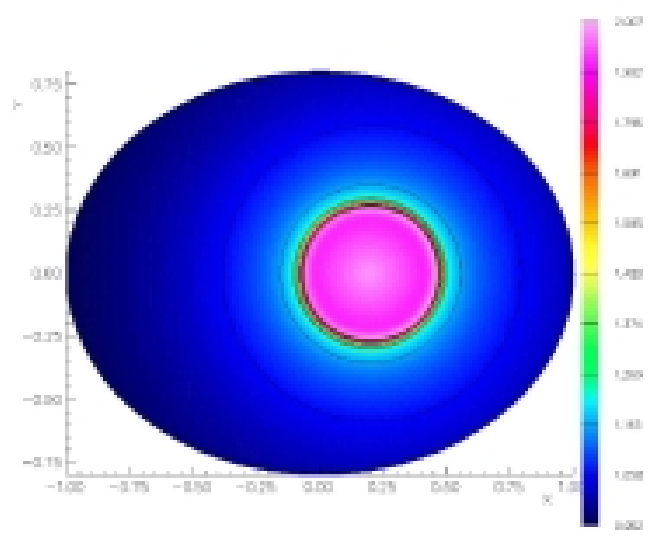

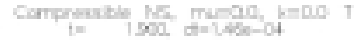

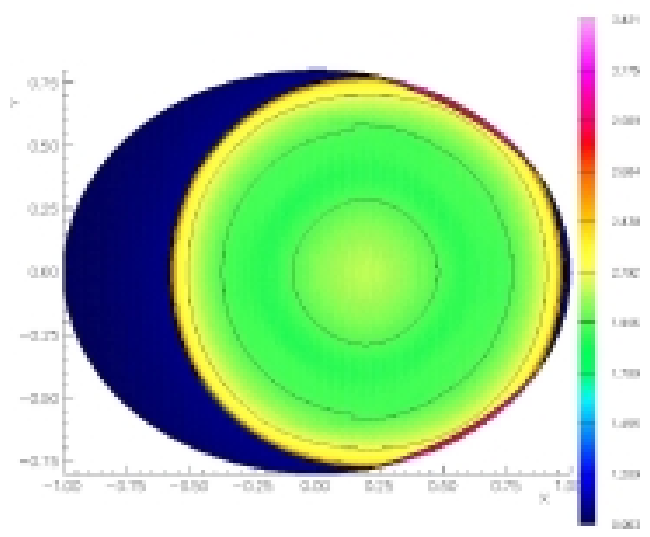

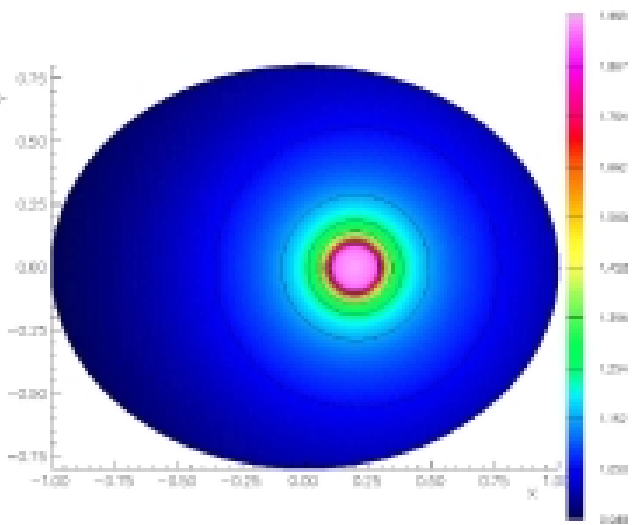

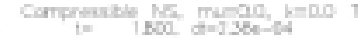

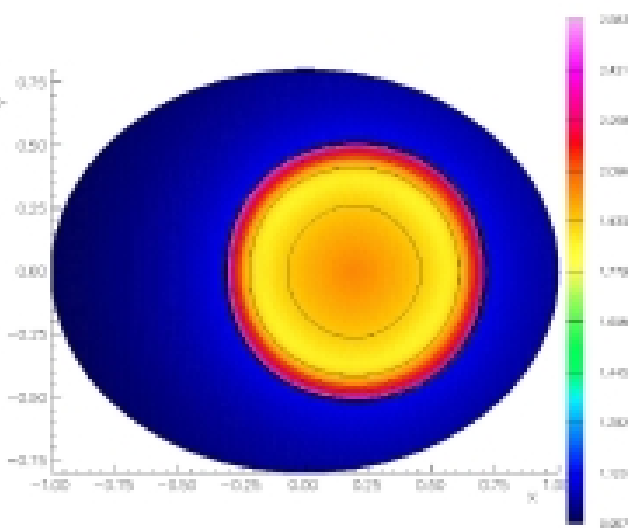

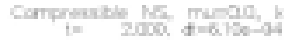

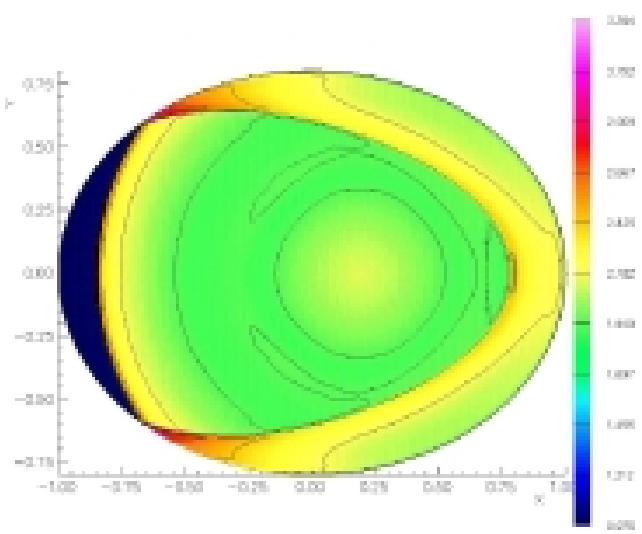

Figure 2: Evolution of the temperature from $t=1.5$ to 2.0 . 

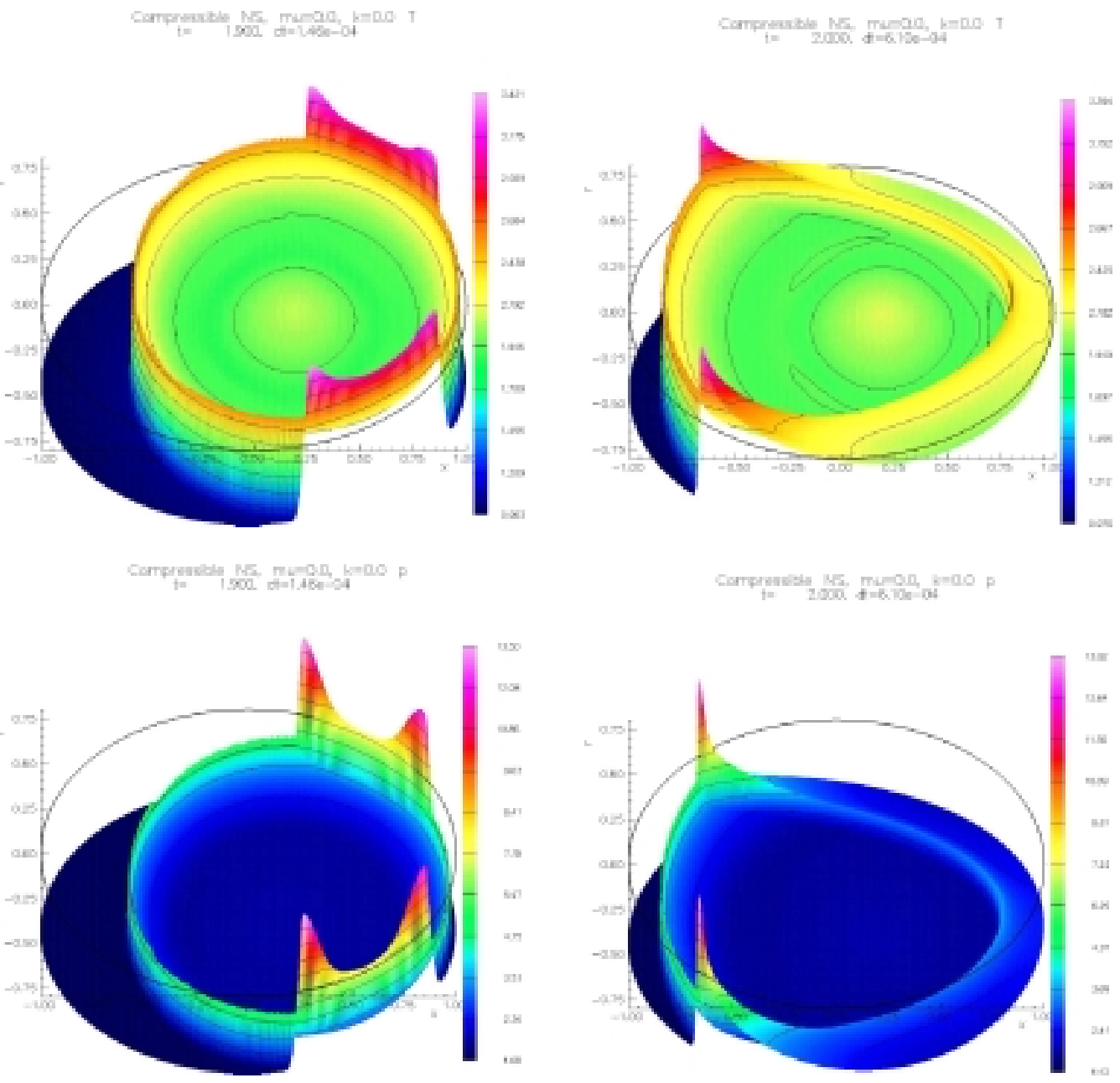

Figure 3: Peak temperatures and pressures during wall interaction.

radially away from the initial hot-spot location and meets the wall at approximately $t=1.9$. At the wall, a reflection occurs creating a high temperature peak. Peak temperatures and pressures during the wall interaction are shown in Figure 3.

It should be noted that while the numerical solution shown in the figures is correct qualitatively, the peak temperatures and pressures are exected to be somewhat lower than the values given by an exact solution of the equations. This is because the grid used is too coarse to fully resolve the reaction zone. There is current work on an AMR implementation of the numerical method which would allow a much finer grid resolution of the reaction zone and thus a more accurate representation of the solution.

\section{Concluding Remarks}

Work on the development of software tools for the numerical solution of high speed 
reactive flow was initiated under the LLNL subcontract. The core elements of the software are now in place and have been tested for accuracy and shown to be useful for numerical simulations. In particular, the software has been used to study the evolution to detonation for a nonuniform reactive sample at critical conditions. While the project has been successful, there are tasks that remain for future work. For example, on the software side, it would be very useful to have an AMR implementation. Work on this aspect of the software is in progress. At present, a Godunov-type numerical flux is implemented with a Roe-type approximate Riemann solver. There are many other choices of numerical flux functions and Riemann solvers that one might use and build into the software as a user option. Additionally, it would be useful to include options for various multiple kinetic reactions, such as chain branching reactions. A long-term goal would be to include diffusive terms in the model equations in order to handle problems involving low-speed flames.

\section{References}

[1] W.D. Henshaw, A Primer for Writing PDE Codes with Overture, LLNL Research Report UCRL-MA-132231, 1998.

[2] W.D. Henshaw, Ogen: An Overlapping Grid Generator for Overture, LLNL Research Report UCRL-MA-132237, 1998.

[3] W.D. Henshaw, OverBlown: A Fluid Flow Solver For Overlapping Grids, User's Guide, LLNL Research Report UCRL-MA-134288, 1999.

[4] W.D. Henshaw, OverBlown: A Fluid Flow Solver For Overlapping Grids, Reference Guide, LLNL Research Report UCRL-MA-134289, 1999.

[5] P. Colella, Multidimensional Upwind Methods for Hyperbolic Conservation Laws, J. Comp. Phys., 87 (1990), pp. 171-200.

[6] P. Glaister, An Approximate Linearised Riemann Solver for the Euler Equations for Real Gases, J. Comp. Phys., 74 (1988), pp. 382-408.

[7] W.D. Henshaw, The plotStuff Graphics Post Processor for Overture, User Guide, Version 1.00, LLNL Research Report UCRL-MA-138730, 1999. 